\title{
ALGEBRAIC SURFACES WITH QUOTIENT SINGULARITIES - INCLUDING SOME DISCUSSION ON AUTOMORPHISMS AND FUNDAMENTAL GROUPS
}

\author{
J. KeUm AND D. -Q. ZhanG
}

\section{INTRODUCTION}

We work over the complex numbers field $\mathbf{C}$. In the present survey, we report some recent progress on the study of varieties with mild singularities like log terminal singularities (which are just quotient singularities in the case of dimension 2; see [KMM]). Singularities appear naturally in many ways. The minimal model program developed by Mori et al shows that a minimal model will inevitably have some terminal singularities [KMo]. Also the degenerate fibres of a family of varieties will have some singularities.

We first follow Iitaka's strategy to divide (singular) varieties $Y$ according to the logarithmic Kodaira dimension $\kappa\left(Y^{0}\right)$ of the smooth locus $Y^{0}$ of $Y$. One key result (2.3) says that for a relatively minimal $\log$ terminal surface $Y$ we have either nef $K_{Y}$ or dominance of $Y^{0}$ by an affine-ruled surface. It is conjectured to be true for any dimension [KMc].

In smooth projective surfaces of general type case, we have Miyaoka-Yau inequality $c_{1}^{2} \leq 3 c_{2}$ and Noether inequalities: $p_{g} \leq(1 / 2) c_{1}^{2}+2, c_{1}^{2} \geq(1 / 5) c_{2}-(36 / 5)$. Similar inequalities are given for $Y^{0}$ in Section 4; these will give effective restriction on the region where non-complete algebraic surfaces of general type exist.

In Kodaira dimension zero case, an interesting conjecture (3.12) (which is certainly true when $Y$ is smooth projective by the classification theory) claims that for a relatively minimal and log terminal surface $Y$ of Kodaira dimension $\kappa\left(Y^{0}\right)=0$, one has either $\pi_{1}\left(Y^{0}\right)$ finite, or an etale cover $Z^{0} \rightarrow Y^{0}$ where $Z^{0}$ is the complement of a finite set in an abelian surface $Z$. Some partial answers to (3.12) are given in Section 3 .

The topology of $Y^{0}$ is also very interesting. We still do not know whether $\pi_{1}$ of the complement of a plane curve is always residually finite or not. Conjecture (2.4) proposed in [Z7] claims that the smooth locus of a log terminal Fano variety has finite topological fundamental group. This is confirmed when the dimension is two and now there are three proofs: [GZ1, 2] (using Lefschetz hyperplane section theorem and Van Kampen theorem), $[\mathrm{KMc}]$ (via rational connectivity), [FKL] (geometric). 
The other interesting topic covered is the automorphism groups. Recent progress in K3 surface case is treated in Section 5. For generic rational surface $X$ of degree $\leq 5$, it is classically known that $|\operatorname{Aut}(X)|$ divides 5 !. However, when $Y$ is a $\log$ terminal del Pezzo singular surface of Picard number 1 , it is very often that $\operatorname{Aut}(Y)$ contains $\mathbf{Z} /(p)$ for all prime $p \geq 5$ (see [Z9] or (6.2)).

\section{TERMinology And Notation}

(1). For a variety $V$ we denote by $V^{0}=V$-Sing $V$ the smooth locus.

(2). A $(-n)$-curve $C$ on a smooth surface is a smooth rational curve with $C^{2}=-n$.

(3). For a divisor $D$, we denote by $\# D$ the number of irreducible components of Supp $D$.

(4). For a variety $V$, the $e(V)$ is the Euler number.

\section{SeCtion 1. Preliminaries}

(1.1). Let $V^{0}$ be a nonsingular variety and let $V$ be a smooth completion of $V^{0}$, i.e., $V$ is nonsingular projective and $D:=V \backslash V^{0}$ is a divisor with simple normal crossings. If $H^{0}\left(V, m\left(K_{V}+D\right)\right)=0$ for all $m \geq 1$, we define the Kodaira (logarithmic) dimension $\kappa\left(V^{0}\right)=-\infty$. Otherwise, $\left|m\left(K_{V}+D\right)\right|$ gives rise to a rational map $\varphi_{m}$ for some $m$ and we define the Kodaira dimension $\kappa\left(V^{0}\right)$ as the maximum of $\operatorname{dim}\left(\varphi_{m}\left(V^{0}\right)\right)$.

The Kodaira dimension of $V^{0}$ does not depend on the choice of the completion $V$ [I3, $\S 11.2]$. Also $\kappa\left(V^{0}\right)$ takes value in $\left\{-\infty, 0,1, \ldots, \operatorname{dim} V^{0}\right\}$.

$V^{0}$ is of general type if $\kappa\left(V^{0}\right)=\operatorname{dim} V^{0}$.

$p_{g}\left(V^{0}\right)=h^{0}\left(V, K_{V}+D\right)$ is called the logarithmic geometric genus which does not depend on the choice of the completion $V$ [ibid.].

(1.2).

(a) Let $G \subseteq G L_{2}$ (C) be a non-trivial finite group with no reflection elements. Then $\mathbf{C}^{2} / G$ has a unique singularity at $\bar{O}$ (the image of the origin of the affine plane $\mathbf{C}^{2}$ ). A singularity $Q$ of a normal surface $Y$ is a quotient singularity if locally the germ $(V, Q)$ is analytically isomorphic to $\left(\mathbf{C}^{2} / G, \bar{O}\right)$ for some $G$. Quotient singularities are classified in [Br, Satz 2.11]. (b) When $\operatorname{dim} Y=2$, the $Q$ in $Y$ is a quotient singularity if and only if it is a log terminal singularity $[\mathrm{Ka} 2$, Cor 1.9].

$Q$ is a $D u$ Val (or rational double, or Dynkin type ADE, or canonical, or rational Gorenstein in other notation) singularity if $G \subseteq S L_{2}(\mathbf{C})$ (see [Du], [Re1]).

In (1.3) - (1.6) below, we assume that $Y$ is a normal projective surface with at worst quotient singularities.

(1.3). Let $f: \widetilde{Y} \rightarrow Y$ be the minimal resolution and $D$ the exceptional divisor. We can write $f^{*} K_{Y}=K_{\tilde{Y}}+D^{*}$ where $D^{*}$ is an effective $\mathbf{Q}$-divisor with support in $D$. Write $D=\sum_{i=1}^{n} D_{i}$ with irreducible $D_{i}$ and $D^{*}=\sum_{2}^{n} d_{i=1} d_{i}$. 


\section{Lemma.}

(1) Each $D_{i}$ is a $\left(-n_{i}\right)$-curve for some $n_{i} \geq 2$.

(2) $0 \leq d_{i}<1$.

(3) $d_{i}=0$ holds if and only if the connected component of $D$ containing $D_{i}$ is contracted to a $\mathrm{Du}$ Val singularity on $Y$ (i.e., $f\left(D_{i}\right)$ is a Du Val singularity).

Proof. (2) follows from the fact that a quotient singularity is just a log terminal singularity [Ka2, Cor 1.9]. For (1) and (3), see [Br, Satz 2.11] and [Ar1, Theorem 2.7].

(1.4). $Y$ is a $\log$ del Pezzo surface if the anti-canonical divisor $-K_{Y}$ is $\mathbf{Q}$-ample. $Y$ is a Gorenstein (log) del Pezzo surface if further $Y$ has at worst Du Val singularities (see [Mi, Ch II, 5.1], [MZ1]).

A normal variety $V$ is Fano if $-K_{V}$ is $\mathbf{Q}$-ample.

A log del Pezzo surface is nothing but a log terminal Fano surface, and a Gorenstein del Pezzo surface is nothing but a canonical Fano surface.

(1.5). $Y$ is a $\log$ Enriques surface if the irregularity $q(Y)=h^{1}\left(Y, \mathcal{O}_{Y}\right)=0$ and if $m K_{Y} \sim 0$ (linear equivalence) for some positive integer $m$. The smallest $m$ is called the index of $Y$ and denoted by $I(Y)$ [Z4, Part I, Definition 1.1].

A $\log$ Enriques surface of index 1 is nothing but a K3 surface possibly with Du Val singularities. A non-rational $\log$ Enriques surface is of index 2 if and only if it is an Enriques surface possibly with Du Val singularities. The case of $Y$ with a unique singularity is classified by Tsunoda [Ts, Proposition 2.2] (see also [Z4, Part I, Proposition 1.6]).

Proposition. Let $Y$ be a rational log Enriques surface with \# $(\operatorname{Sing} Y)=1$. Then $I(Y)=2$ and the unique singularity is of type $(1 / 4 n)(1,2 n-1)$ for some $n \geq 1$.

(1.6). The surface $Y$ is relatively minimal if for every curve $C$, we have either $K_{Y} . C \geq 0$ or $C^{2} \geq 0$.

Suppose that $Y$ is not relatively minimal. Then there is a curve $C$ such that $K_{Y} . C<0$ and $C^{2}<0$. By [MT1, Lemma $1.7(2)$ ], we see that there is a contraction $Y \rightarrow Z$ of the curve $C$ to a smooth or quotient singularity such that the Picard number $\rho(Z)=\rho(Y)-1$. So every projective surface with at worst quotient singularities has a relatively minimal model.

$Y$ is strongly minimal if it is relatively minimal and if there is no curve $C$ with $C^{2}<0$ and $C . K_{Y}=0[\mathrm{Mi}$, Ch II, (4.9)].

(1.7). A smooth projective rational surface $X$ is a Coble surface if $\left|-K_{X}\right|=\emptyset$ while $\left|-2 K_{X}\right| \neq \emptyset$. A Coble surface is terminal if it is not the image of any birational but not biregular morphism of Coble surface.

Coble surfaces are classified in [DZ]. Here is an example. Let $Z$ be a rational elliptic 
surface with a multiplicity-2 fibre $F_{0}$ and a non-multiple fibre $F_{1}$ of type $I_{n}$ (see [CD] for classification of $Z$ ). Let $X \rightarrow Z$ be the blow up of all $n$ intersection points in $F_{1}$. Then $X$ is a terminal Coble surface. Coble surfaces and $\log$ Enriques surfaces are closely related.

Proposition [DZ, Proposition 6.4].

(1) The minimal resolution $X$ of a rational log Enriques surface $Y$ of index 2 is a Coble surface with $h^{0}\left(X,-2 K_{X}\right)=1$ and the only member $D$ in $\left|-2 K_{X}\right|$ is reduced and a disjoint union of $D_{i}$, where $D_{i}$ is either a single $(-4)$-curve or a linear chain with the dual graph below (each $D_{i}$ is contractible to a singularity of type $\left(1 / 4 n_{i}\right)\left(1,2 n_{i}-1\right)$ with $\left.n_{i}=\# D_{i}\right)$ :

$$
(-3)--(-2)--\cdots--(-2)--(-3) \text {. }
$$

If we let $X_{t e} \rightarrow X$ be the blow up of all intersection points in $D$, then $X_{\text {te }}$ is a terminal Coble surface with $h^{0}\left(X_{t e},-2 K_{X_{t e}}\right)=1$ and the only member in $\left|-2 K_{X_{t e}}\right|$ is a disjoint union of $n$ of $(-4)$-curves with $n=\sum_{i} n_{i}$.

(2) Conversely, a terminal Coble surface $X$ has a unique member $D$ in $\left|-2 K_{X}\right|$, and $D$ is reduced and a disjoint union of (-4)-curves.

(1.8). A smooth affine surface $S$ is a $\mathbf{Q}$-homology plane if $H_{i}(S, \mathbf{Q})=0$ for all $i>0$. Similarly we can define a $\mathbf{Z}$-homology plane and $\mathbf{Q}$-homology plane with quotient singularities. The following very important theorem is proved by Gurjar, Pradeep and Shastri in their papers [GS], [PS], [GPS] and GPr].

Theorem 1.9. A Q-homology plane with at worst quotient singularities is a rational surface.

Theorem 1.10 [Mi, Theorem 4.10]. Let $Y$ be a $\mathbf{Q}$-homology plane. Let $\nu$ be the number of topologically contractible curves in $Y$. Then we have:

(1) Every topologically contractible curve is isomorphic to the affine line.

(2) The Kodaira dimension $\kappa\left(Y^{0}\right)=2,1$ or $0,-\infty$ if and only if $\nu=0$, finite, $\infty$, respectively. (Gurjar and Parameswaran [GPa] have determined the number $\nu$ when $\kappa\left(Y^{0}\right)=0$ ). (3) Suppose that $Y$ is a homology plane. Then $\kappa\left(Y^{0}\right)=2,1,-\infty$ if and only if $\nu=0,1, \infty$, respectively (see (3.17)).

\section{Section 2. Normal Algebraic Surfaces $Y$ with KodAIRA DIMENSION $\kappa\left(Y^{0}\right)=-\infty$ AND FANO VARIETIES}

In this section we consider projective varieties with at worst log terminal singularities and Kodaira dimension $\kappa\left(Y^{0}\right)=-\infty$, where $Y^{0}=Y-\operatorname{Sing} Y$.

The following result is a special case of [MT1, Theorem 2.11]. We will sketch a different and direct proof here by making use of $[\mathrm{KMM}]$.

Theorem 2.1. Let $Y$ be a relatively minimal surface with at worst quotient singularities. Then one of the following occurs. 
(1) The Kodaira dimension $\kappa\left(Y^{0}\right) \geq 0$ and $K_{Y}$ is numerically effective.

(2) $\kappa\left(Y^{0}\right)=-\infty$ and $K_{Y}$ is not numerically effective. To be precise, either

(2a) $Y^{0}$ is ruled, i.e. $Y^{0}$ has a Zariski open set of the form $\mathbf{P}^{1} \times C$ with a curve $C$, or

(2b) $Y$ is a log del Pezzo surface of Picard number 1.

Proof. We may assume that $K_{Y}$ is not nef. Then by [KMM, Theorems 4-2-1 and 3-2-1], there is an extremal ray $\mathbf{R}_{>0}[C]$ with $C$ a rational curve, and a corresponding morphism $\Phi: Y \rightarrow Z$ with connected fibres such that a curve $E$ is mapped to a point by $\Phi$ if and only if the class of $E$ is in $\mathbf{R}_{>0}[C]$.

Case $\operatorname{dim} Z=2$. Then $Z$ has at worst $\log$ terminal singularities (= quotient singularities) by [KMM, Proposition 5-1-6]. This contradicts the relative minimality of $Y$.

Case $\operatorname{dim} Z=0$. Then Pic $Y$ is generated over $\mathbf{Q}$ by $C$ and hence Picard number $\rho(Y)=1$ and $C$ is $\mathbf{Q}$-ample. Since $K_{Y} . C<0$, we have $K_{Y}=a C$ (numerically) with $a<0$. So the case $(2 \mathrm{~b})$ occurs.

Case $\operatorname{dim} Z=1$. Then a general fibre $F$ of $\Phi$ is $\mathbf{P}^{1}$ because $F^{2}=0$ and $K_{Y} \cdot F<0$ (pull back to $\widetilde{Y}$ and use genus formula). Clearly the case (2a) occurs. This proves the theorem.

Theorem 2.2 ([KMc, Cor. 1.6], [Mi, Ch II, Theorems 2.1 and 2.17]).

Let $Y$ be a log del Pezzo surface. Then there is a dominant morphism $X^{0} \rightarrow Y^{0}$ such that $X^{0}$ is an affine-ruled surface (i.e., $X^{0}$ contains a Zariski open set of the form $\mathbf{A}^{1} \times C$ for some curve $C$ ).

When $Y$ is Gorenstein, Theorem 2.2 was proved in [Z2, Theorem 3.6]; the general case of Theorem 2.2 was proved in a lengthy book [KMc, Cor 1.6]; Theorems 2.1 and 2.2 together give the proof of the following result, which is the quotient surface case of Miyanishi Conjecture.

Theorem 2.3. Let $Y$ be a projective surface with at worst quotient singularities. Suppose that $Y$ is relatively minimal. Then the following are equivalent.

(1) $K_{Y}$ is not nef.

(2) $\kappa\left(Y^{0}\right)=-\infty$.

(3) There is a dominant morphism $X^{0} \rightarrow Y^{0}$ such that $X^{0}$ is an affine-ruled surface.

Proof. The equivalence of (1) and (2) is proved in [MT1, Theorem 2.11]. (2) implies (3) by Theorems 2.1 and 2.2. Assume (3). Now $\kappa\left(X^{0}\right)=-\infty$ is clear by considering a ruled surface as a completion of $X^{0}$ with the boundary equal to the union of a section (or empty set) and a few fibre components. Since $\kappa\left(X^{0}\right) \geq \kappa\left(Y^{0}\right),(2)$ follows.

Now we turn to the topology of smooth locus of a variety. We proposed the following 
in [Z7].

Conjecture 2.4. Let $V$ be a Fano variety with at worst log terminal singularities. Then the topological fundamental group $\pi_{1}\left(V^{0}\right)$ is finite.

The affirmative answer to (2.4) would imply the following which was conjectured in [KZ] and is now a theorem of S. Takayama [Ta]. Indeed, (2.4) would imply that $\pi_{1}(V)$ is finite and we let $U \rightarrow V$ be the universal cover. Then $\chi\left(\mathcal{O}_{U}\right)=n \chi\left(\mathcal{O}_{V}\right)$, where $n=\left|\pi_{1}(V)\right|$. The Kawamata-Viehweg vanishing implies that $\chi\left(\mathcal{O}_{X}\right)=h^{0}\left(X, \mathcal{O}_{X}\right)(=1)$ for both $X=U$ and $V$. Hence $n=1$.

Theorem 2.5. Let $V$ be a Fano variety with at worst log terminal singularities. Then $\pi_{1}(V)=(1)$.

The result (2.5) would also follow from the following conjecture which is still open for dimension 4 or higher. It is proved in 3 -fold case by [Ca] and [KoMiMo]. Recently, Graber, Harris and Starr [GHS] have proved that any complex algebraic variety having a fibration with rationally connected general fibres and image (or base), is again rationally connected.

Conjecture 2.6. Let $V$ be a Fano variety with log terminal singularities. Then $V$ is rationally connected, i.e., any two general points are connected by an irreducible rational curve.

Partial answers to $(\mathbf{2 . 4})$ are given in $(\mathbf{2 . 7}) \sim(\mathbf{2 . 1 0})$.

(2.7). When dimension is 2, Conjecture 2.4 was proved in affirmative by [GZ1, 2]; for a differential geometric proof, see [FKL]. In [KMc, Cor 1.6], it was proved that for a log del Pezzo surface $Y$, the $Y^{0}$ is rationally connected and hence has finite $\pi_{1}\left(Y^{0}\right)$ (see [Ca] and [KoMiMo]).

Theorem 2.8 [Z7, Theorem 2].

Conjecture 2.4 is true if one of the following occurs.

(1) $\operatorname{dim} V \leq 2$.

(2) The Fano index $r(V)>\operatorname{dim} V-2$.

(3) $V$ has only isolated singularities and $r(V)=\operatorname{dim} V-2=1$.

Theorem 2.9 [Z7, Theorem 2].

Let $V$ be a Fano variety of Fano index $r(V)>\operatorname{dim} V-2$ and with at worst canonical singularities. Then $\pi_{1}\left(V^{0}\right)$ is abelian of order $\leq 9$.

Theorem 2.10 [Z7, Theorems 1 and 2].

Let $V$ be a Fano variety. Then $\pi_{1}\left(V^{0}\right)=(1)$ if one of the following occurs: 
(1) The Fano index $r(V)>\operatorname{dim} V-1$.

(2) $\operatorname{dim} V=3$ and $V$ has only Gorenstein isolated singularities.

(2.11). The following gives a concrete upper bound for $\pi_{1}\left(V^{0}\right)$ in certain case. A relation $m\left(K_{V}+H\right) \sim 0$ in the theorem below occurs when $V$ has Fano index 1 and Cartier index $m$. It is conjectured that $m=1,2$. To prove the theorem below, we show first that there is a natural surjective map $\pi_{1}\left(H^{0}\right) \rightarrow \pi_{1}\left(V^{0}\right)$ and also use the fact that $H$ is Du Val K3 or Enriques. Now the theorem follows from the results on $H$ in [KZ, Theorems 1 and 2]. For each of the three exceptional cases of $(p, c)$ below, we note that there is a Du Val K3 or Enriques surface $Y$ with $\operatorname{Sing} Y=c A_{p-1}$ and $\pi_{1}\left(Y^{0}\right)$ infinity [ibid.].

Theorem [KZ, Theorem 3].

Let $p$ be a prime number. Let $V$ be a log terminal Fano 3-fold with a Cartier divisor $H$ such that $m\left(K_{V}+H\right) \sim 0$ (linear equivalence) for $m=1$ or 2 . Suppose that a member $H$ of $|H|$ is irreducible normal and has $c$ singularities of type $A_{p-1}$ and no other singularities. Then $\pi_{1}\left(V^{0}\right)$ is soluble; and if $(p, c) \neq(2,8),(2,16),(3,9)$, then $\left|\pi_{1}\left(V^{0}\right)\right| \leq 2 p^{k}$ for some $k \leq 4$.

\section{Remark 2.12.}

(1) In (2.4) if we replace "log terminal" by "log canonical", then (2.4) has counterexamples; more precisely, if $V$ is a normal Fano surface with at worst rational log canonical singularities, then $\pi_{1}\left(V^{0}\right)$ contains a finite-index abelian subgroup of rank $k(k=0,2)$ [Z8, Theorem 2.3].

(2) In (2.9), the upper bound is optimum [MZ1, Lemma 6]; also "canonical" can not be replaced by "log terminal" [Z3, Appendix].

(2.13). In [Kj2], log del Pezzo surfaces with a unique singularity are classified (including the existence part). The classification of log del Pezzo surface of Cartier index $\leq 2$ were announced in [AN]. In [Kj4], Kojima classified Picard number $1 \log$ del Pezzo surfaces $Y$ of index 2, in a way different from [AN]: there are exactly 18 types of $\operatorname{Sing} Y$ and the $\pi_{1}\left(Y^{0}\right) \leq 8$; the $\pi_{1}\left(Y^{0}\right)=(1)$ holds if and only if $Y$ contains the affine plane as a Zariski open set.

(2.14). In [Ni3], the Picard number $\rho(\tilde{Y})$ of the minimal resolution $\widetilde{Y}$ of a log del Pezzo surface $Y$ is bounded from above in terms of the maximum of multiplicities of $Y$.

\section{Section 3. Normal algebraic surfaces $Y$ with Kodaira dimension $\kappa\left(Y^{0}\right)=0$}

In this section we consider projective surfaces $Y$ with at worst quotient singularities and Kodaira dimension $\kappa\left(Y^{0}\right)=0$, where $Y^{0}=Y-\operatorname{Sing} Y$.

Theorem 3.1 ([Ka1, Theorem 2.2], [Mi, Ch II, 6.1.3]). 
Let $Y$ be a projective surface with at worst quotient singularities. Then the following are equivalent:

(1) $Y$ is relatively minimal with Kodaira dimension $\kappa\left(Y^{0}\right)=0$.

(2) There is a positive integer $m$ such that $m K_{Y} \sim 0$ (linear equivalence).

(3.2). The smallest positive integer $m$ with $m K_{Y} \sim 0$ is called the index of $Y$ and denoted by $I=I(Y)$.

Proposotion 3.3. Let $Y$ be a projective surface with at worst quotient singularities and $I K_{Y} \sim 0$, where $I>0$ is the index of $Y$. Suppose that $Y$ is irrational. Then one of the following occurs.

(1) $Y$ is a (smooth) abelian surface $(I=1)$ or a hyperelliptic surface $(I=2,3,4,6)$.

(2) $Y$ has at worst Du Val singularities. The minimal resolution of $Y$ is either a K3 surface $(I=1)$ or an Enriques surface $(I=2)$.

Proof. In notation of (1.3), we have $I\left(K_{\tilde{Y}}+D^{*}\right) \sim 0$. So $\kappa(\widetilde{Y}) \leq 0$. If $\kappa(\widetilde{Y})=0$, Then (3.3) follows from the classification of smooth surfaces. If $\kappa(\tilde{Y})=-\infty$, then $\widetilde{Y}$ is an irrational ruled surface over a base curve of genus $\geq 1$. However, $K_{\widetilde{Y}}+D^{*}=0$ (numerically) implies that $D^{*}$ contains some horizontal components (this can be seen by going to a relative minimal model of $\widetilde{Y}$ ) which dominates the base curve and hence is irrational, contradicting the fact that $D$ consists of rational curves only (1.3). So $\kappa(\tilde{Y})=-\infty$ is impossible. This proves the proposition.

In view of (3.3), to classify those $Y$ with at worst quotient singularities and $\kappa\left(Y^{0}\right)=0$, we need only to consider rational surfaces $Y$ with $m K_{Y} \sim 0$ for some integer $m \geq 2$ (see (1.5)). These are precisely rational log Enriques surfaces (1.5).

(3.4). Let $Y$ be a rational $\log$ Enriques surface. Then the index $I=I(Y) \geq 2$. Since $I K_{Y} \sim 0$, there is a canonical $\mathbf{Z} /(I)$-Galois cover $\pi: X=\operatorname{Spec} \oplus_{j=0}^{I-1} \mathcal{O}\left(-j K_{Y}\right) \rightarrow Y$ which is unramified over $\left(Y \backslash\right.$ non-Du Val singularities\}) $\supseteq Y^{0}$ and satisfies $K_{X} \sim 0$. Therefore, either $X$ is a (smooth) abelian surface or a K3 surface possibly with some Du Val singularities.

Recently, Suzuki $[\mathrm{Su}]$ has proved Morrison's cone conjecture for rational log Enriques surfaces $Y$ : there is a finite rational polyhedral cone which is a fundamental domain for the action of $\operatorname{Aut}(Y)$ on the rational convex hull of its ample cone.

Theorem 3.5. Suppose that $Y$ is a rational log Enriques surface of index $I$ and that the canonical $\mathbf{Z} /(I)$-cover $X$ of $Y$ is an abelian surface. Then we have:

(1) $I=3$ or 5 . If $I=3$, then $\operatorname{Sing} Y$ consists of 9 singularities of type $(1 / 3)(1,1)$; if $I=5$, then Sing $Y$ consists of 5 singularities of type $(1 / 5)(1,2)$; see [Re1] for notation.

(2) For each $I=3,5$, there is a unique log Enriques surface $Y_{I}$ with $X_{I}$ abelain and 
$I\left(Y_{I}\right)=I$. To be precise, $Y_{I}=X_{I} /\left\langle g_{I}\right\rangle$, where $X_{3}=E_{\zeta_{3}} \times E_{\zeta_{3}}$ with $E_{\zeta_{3}}=\mathbf{C} /\left(\mathbf{Z}+\mathbf{Z} \zeta_{3}\right)$ an elliptic curve of period $\zeta_{3}=\exp (2 \pi \sqrt{-1} / 3), g_{3}=\operatorname{diag}\left(\zeta_{3}, \zeta_{3}\right), X_{5}$ is the Jacobian surface of the genus- 2 curve : $y^{2}=x^{5}-1$, and $g_{5}$ in $\operatorname{Aut}\left(X_{5}\right)$ is induced by the curve automorphism $:(x, y) \mapsto\left(\zeta_{5} x, y\right)$ (see [Bl, Example 1.2], [Su, Proposition 1.2] and [Z4, Example 4.2]).

Proof. (1) is proved in [Z4, Theorem 4.1]. (2) is proved in [Bl, Su].

Theorem 3.6. Let $Y$ be a log Enriques surface of index $I$. Then $I \leq 21$.

\section{Remark 3.7.}

(1) It is easy to see that the Euler function $\varphi(I) \leq 21$ and hence $I \leq 66$ [Z4, Part I, Lemma 2.3]. In [Bl, Theorem $\mathrm{C}]$, it is proved that $I \leq 21$.

(2) Examples of $Y$ with prime $I(Y)$ are constructed in [Z4, Example 5.3-5.8; Part II, Example 7.3] and [Bl, Example 4.1].

(3.8). A $\log$ Enriques surface $Y$ is maximum if any birational morphism $Y \rightarrow Z$ to another log Enriques surface is an isomorphism. By [Z4, Part II, Theorem 2.11'], for every $\log$ Enriques surface $Y$ of prime index, there is a unique maximum $\log$ Enriques surface $Y_{\max }$ with $I\left(Y_{\max }\right)=I(Y)$ and a birational morphism $Y_{\max } \rightarrow Y$; each singularity (if exists) of the canonical cover of $Y_{\max }$ is of type $A_{1}$.

The surface $Y\left(A_{19}\right)$ in the assertion(3) below is not isomorphic to the unique (modulo projective transformation) quartic $K 3$ surface in $\mathbf{P}^{3}$ with a Dynkin type $A_{19}$ singularity; neither can $Y\left(D_{19}\right)$ be embedded in $\mathbf{P}^{3}[\mathrm{KN}]$. The $Y\left(D_{19}\right)$ is constructed in two different ways in [Z4, Example 6.11: the $\left.\bar{V}^{\prime}\right]$ and [OZ1, Example 1], and $Y\left(A_{19}\right)$ in [Z4, Example 3.2 : the $\bar{V}$ ] and [OZ1, Example 2]. The uniqueness problem of $Y\left(D_{19}\right)$ was initiated by [Re2, Round 3, Example 6].

Theorem [OZ4, Corollary 4; OZ3, Corollary in $\S 1$; OZ1, Theorems 1 and 2].

(1) For each $I=13,17,19$, there is a unique maximum log Enriques surface $Y$ with $I(Y)=$ $I$.

(2) All maximum log Enriques surfaces of index 11 form a family of dimension 1 and are all given in [OZ3, $\S 1$, Corollary].

(3) For each $D$ in $\left\{D_{19}, A_{19}\right\}$, there is a unique rational log Enriques surface $Y(D)$ whose canonical cover has a singularity of Dynkin type D. The index $I(Y)$ equals 3 (resp. 2) when $Y$ equals $Y\left(D_{19}\right)$ (resp. $Y\left(A_{19}\right)$ ).

Next we will investigate the behaviour of $\pi_{1}\left(Y^{0}\right)$ and propose a conjecture (3.12) generalizing the one in [CKO]. Note that $\mathbf{Z} /(I)$ is the image of $\pi_{1}\left(Y^{0}\right)$ by a homomorphism.

Theorem 3.9 [Z4, Part II, Theorem 2.11', Cor 1]. Let $Y$ be a maximum log Enriques surface of odd prime index $I$. Let $X \rightarrow Y$ be the canonical $\mathbf{Z} /(I)$-cover. Then we have:

(1) $X$ has at worst type $A_{1}$ singularities and $\#(\operatorname{Sing} X) \leq 6$. 
(2) $\pi_{1}\left(Y^{0}\right)=\mathbf{Z} /(I)$.

Proof. (1) is proved in [Z4, Part II, Cor. 1]. For (2), we have only to show that $\pi_{1}\left(X^{0}\right)=(1)$ because the inverse of $Y^{0}$ via the canonical map $X \rightarrow Y$ (unramified over $Y^{0}$ ) is $X^{0}$ with a few smooth points removed (the removal of smooth points in a complex surface does not change $\left.\pi_{1}\right)$. Since $\#(\operatorname{Sing} X) \leq 6$ by $(1)$, we have $\pi_{1}\left(X^{0}\right)=(1)[\mathrm{KZ}$, Theorem 1]. This proves the theorem.

Theorem 3.10 [SZ, Proposition 4.1, Theorem 4.3, Cor 4.4]

(1) Suppose that " $\pi_{1}^{\text {alg }}\left(X^{0}\right)=(1) \Rightarrow \pi_{1}\left(X^{0}\right)=(1)$ for all Du Val K3 surfaces $X "$ holds ( $X$ satisfies this condition if $(*)$ in (2) holds for $X$ ). Let $Y$ be a $\log$ Enriques surface of index $I$. Then either $\pi_{1}\left(Y^{0}\right)$ is finite or there is a finite morphism $Z \rightarrow Y$ from an abelian surface which is unramified over $Y^{0}$. In particular, $\pi_{1}\left(Y^{0}\right)$ contains a finite-index abelian subgroup of rank $k(k=0,4)$.

(2) Let $X \rightarrow Y$ be the canonical cover of a log Enriques surface of index $I$, let $\widetilde{X} \rightarrow X$ be the minimal resolution and $D=\sum D_{i}$ the exceptional divisor. Then $\pi_{1}\left(Y^{0}\right)=\mathbf{Z} /(I)$ if the lattice $\Gamma=\mathbf{Z}\left[\cup D_{i}\right]$ is primitive in $H^{2}(\widetilde{X}, \mathbf{Z})$ and satisfies:

$\left.{ }^{*}\right) r=\operatorname{rank}(\Gamma) \leq 18$ and the discriminant group $\Gamma^{\vee} / \Gamma$ is generated by $k$ elements with $k \leq \min \{r, 20-r\}$.

Proof. Let $X \rightarrow Y$ be the canonical $\mathbf{Z} /(I)$-cover. Now the conclusion in (3.10) (1) with $Y$ replaced by $X$ holds by [SZ, Proposition 4.1]. So (1) is true for $X \rightarrow Y$ is unramified over $Y^{0}$. For (2), [SZ, Theorem 4.3] implies $\pi_{1}\left(X^{0}\right)=(1)$. So $(2)$ is true. This proves the theorem.

There is a concrete upper bound of $\pi_{1}\left(Y^{0}\right)$ for certain $Y$.

Theorem 3.11 [GZ3, Theorem 1]. Let $Y$ be a rational log Enriques surface of index 2 . Assume that $Y$ has no Du Val singularities. Then $\pi_{1}\left(Y^{0}\right)$ is a soluble group of order $n_{1} n_{2}$ with $n_{i} \leq 16$.

The results $(3.9) \sim(3.11)$ support the following which is just the conjecture in [CKO] when $Y$ is a Du Val $K 3$ surface, i.e., when $I(Y)=1$ and $q(Y)=0$.

Conjecture 3.12. Let $Y$ be a log Enriques surface. Then the universal cover $U$ of $Y^{0}$ is a big open set (= the complement of a discrete subset) of either a Du Val K3 surface or of $\mathbf{C}^{2}$; in the latter case, $U \rightarrow Y^{0}$ factors through a finite etale cover $Z^{0} \rightarrow Y^{0}$, where $Z^{0}$ is a big open set of an abelian surface $Z$.

(3.13). Since the canonical cover $X \rightarrow Y$ of a $\log$ Enriques surface is unramified over $Y^{0}$, we have $\pi_{1}\left(Y^{0}\right) / \pi_{1}\left(X^{0}\right)=\mathbf{Z} /(I)$. So (3.12) is, in most cases, reduced to the problem of $\pi_{1}\left(X^{0}\right)$ for a Du Val K3 surface $X$ (see (3.5)). We have the following: 
Theorem [KZ, Theorems 1 and 2]. Conjecture 3.12 is true if $Y$ is a Du Val K3 or Enriques surface and has several singularities of type $A_{p-1}$ and no other singularities; here $p$ is a prime number.

(3.14). The following results contributes towards an answer in affirmative to (3.12). These are just applications of [CKO, Theorems A and B] to the canonical cover $X$ of $Y$. Also the upper bound $\# D \leq 15$ is an optimum condition for $\pi_{1}\left(X^{0}\right)$ to be finite by considering Kummer surfaces.

Theorem. (1) Let $Y$ be a log Enriques surface with an elliptic fibration. Then either $\pi_{1}\left(Y^{0}\right)$ is finite or there is a finite cover of $Z \rightarrow Y$ from an abelian surface which is unramified over $Y^{0}$.

(2) Let $Y$ be a $\log$ Enriques surface, $X \rightarrow Y$ the canonical cover and $\widetilde{X} \rightarrow X$ the minimal resolution with $D$ the exceptional divisor. Suppose that $\# D \leq 15$. Then $\pi_{1}\left(Y^{0}\right)$ is finite.

(3.15). In $[\mathrm{Oh}]$, pairs $(S, \Delta)$ of normal surface $S$ and a Q-divisor $\Delta$ satisfying $K_{S}+\Delta \equiv 0$ (numerically) are considered. These pairs appear naturally as degenerate fibres in log degeneration; for many interesting cases, he completed the classification of these pairs.

(3.16). In [Kj1, Theorem 0.1], strongly minimal smooth affine surface $S$ with $\kappa(S)=0$ is classified and its invariants are classified (strongly minimal means almost minimal and having no exceptional curve of the second kind [Mi, Ch II, (4.9)]). In particular, the minimal $m>0$ with log pluri-genus $P_{m}(S)>0$, the $\log$ irregularity $q(S)$ and the Euler number $e(S)$ satisfy the following $\left(\pi_{1}(S)\right.$ is also calculated there, which is generated by at most two elements):

$$
m \mid 6, \quad q(S) \in\{0,1,2\}, \quad e(S) \in\{0,1,2,3,4\} .
$$

(3.17). In [Fu, §8], all Q-homology planes of Kodaira dimension 0 are classified. It was also proved there that there is no Z-homology plane $S$ of Kodaira dimension $\kappa(S)=0$. The paper $[\mathrm{Fu}]$ is very important and also essentially used in $[\mathrm{Kj} 1]$.

(3.18). Iitaka [I2] conjectured that an affine normal variety $S$ is isomorphic to $\left(\mathbf{C}^{*}\right)^{n}$ if and only if $\kappa(S)=0$ and $q(S)=\operatorname{dim} S$. In the same paper, he himself proved it when $\operatorname{dim} S=2$.

According to [I1], a (possibly open) surface $S$ is logarithmic $K 3$ if the logarithmic invariants satisfy : $q(S)=0, p_{g}(S)=1, \kappa(S)=0$. In [I1] log K3 surfaces were classified. In [Z1], one defines the Iitaka surface as a pair $(V, A+N)$ of smooth projective rational surface $V$ and reduced divisor $A+N$ with $A+K_{V} \sim 0$ and $N$ contractible to Du Val singularities, and the classification of such pairs were done there. 
(3.19). In [Kj3], Kojima studies complements $S$ of reduced plane curves with $\kappa(S)=0$; in particular he proves that the logarithmic geometric genus $p_{g}(S)=1$.

Section 4. Normal algebraic surfaces $Y$ with Kodaira dimension $\kappa\left(Y^{0}\right)=1,2$

In this section we consider projective surface $Y$ with at worst quotient singularities and $\kappa\left(Y^{0}\right)=1,2$.

(4.1). We first consider the case $\kappa\left(Y^{0}\right)=1$. The following is a consequence of [Ka1, Theorem 2.3] or [Mi, Ch II, Theorem 6.1.4]. Indeed, in our case, the boundary divisor $D^{*}$ is fractional and contains no effective integral divisor (1.3).

Theorem. Let $Y$ be a projective surface with at worst quotient singularities. Suppose that $Y$ is relatively minimal and $\kappa\left(Y^{0}\right)=1$.

Then there is a positive integer $m$ such that $m K_{Y}$ is Cartier and the linear system $\left|m K_{Y}\right|$ is composed with an irreducible pencil $\Lambda$ without base points. Each general member of $\Lambda$ is a smooth elliptic curve. So there is an elliptic fibration $Y \rightarrow B$.

(4.2). Let $Y$ be a projective surface with at worst quotient singularities. Suppose that $Y$ is relatively minimal and $\kappa\left(Y^{0}\right)=2$. Then $K_{Y}$ is nef and big. By [KMM, Theorem 3-1-1], $\left|m K_{Y}\right|$ is base point free for $m$ sufficiently divisible, and hence defines a birational morphism $\varphi: Y \rightarrow Z$. This $\varphi$ is nothing but the contraction of all curves on $Y$ having zero intersection with $K_{Y}$. Then $Z$ has at worst log canonical singularities ([Ka1, Theorem 2.9], [Mi, Ch II, Theorem 4.12]). Denote by $L C$ the set of points on $Z$ which is $\log$ canonical but not $\log$ terminal (i.e., not of quotient singularity). Then we have the following Miyaoka-Yau type inequality proved by $[\mathrm{Kb} 1,2]$ and $[\mathrm{KNS}]$.

Theorem 4.3. Let $Y$ be a projective surface with at worst quotient singularities. Suppose that $Y$ is relatively minimal and $\kappa\left(Y^{0}\right)=2$. Then we have the following, where $P$ runs over all quotient singularities of $Z$ and $G_{P}$ is the local fundamental group at $P$

$$
K_{Y}^{2} \leq 3\left\{e(Z-L C)-\sum_{P}\left(1-\frac{1}{\left|G_{P}\right|}\right)\right\}
$$

(4.4). For smooth and minimal surfaces $X$ of general type, we have Noether inequalities

$$
p_{g}(X) \leq(1 / 2) c_{1}(X)^{2}+2, \quad c_{1}(X)^{2} \geq \frac{1}{5} c_{2}(X)-\frac{36}{5} .
$$

For singular surfaces, we have:

Theorem [TZ, Theorems 1.3 and 2.10]. 
Let $Y$ be a projective surface with at worst quotient singularities and $\kappa\left(Y^{0}\right)=2$. Then the logarithmic geometric genus $p_{g}\left(Y^{0}\right)$ satisfies the optimum upper bound:

$$
p_{g}\left(Y^{0}\right)<K_{Y}^{2}+3 .
$$

(4.5). For smooth projective surface $X$ of general type, the famous Miyaoka-Yau inequality asserts that $c_{1}(X)^{2} \leq 3 c_{2}(X)$.

Consider $\log$ surface $(V, D)$ with $V$ a smooth projective surface and $D$ a reduced divisor with simply normal crossings. Set $\bar{c}_{1}^{2}=\left(K_{V}+D\right)^{2}$ and $\bar{c}_{2}=c_{2}(V)-e(D)$. Sakai [Sa] proved that

$$
\bar{c}_{1}^{2} \leq 3 \bar{c}_{2}
$$

provided that $D$ is semi-stable and $\kappa(V \backslash D)=2$. The following is a lower bound of $\bar{c}_{1}^{2}$ in terms of $\bar{c}_{2}$. These two inequalities together give effective restrictions on the region for non-complete algebraic surfaces $V \backslash D$ of general type to exist. In the following, $(V, D)$ is minimal if $K_{V}+D$ is nef and there is no $(-1)$-curve $E$ with $E .\left(K_{V}+D\right)=0$.

Theorem [Z5, Cor. to Theorem $\mathrm{C}$, Theorem $\mathrm{D}]$. Let $(V, D)$ be a log surface with $D \neq 0$. Assume that $(V, D)$ is minimal and $\kappa(V \backslash D)=2$. Assume further that $\kappa(V) \geq 0$. Then we have

$$
\bar{c}_{1}^{2} \geq \frac{1}{15} \bar{c}_{2}-\frac{8}{5} .
$$

(2) Suppose that $p_{g}(V \backslash D) \geq 3$ and $\left|K_{V}+D\right|$ is not composed with a pencil. Then

$$
\bar{c}_{1}^{2} \geq \frac{1}{9} \bar{c}_{2}-2 .
$$

\section{Section 5. Automorphisms of Algebraic surfaces - Smooth Surface Case}

(5.1). We mention some background of $\operatorname{Aut}(X)$ where $X$ is a smooth projective rational surface. Aut $(X)$ had been studied by S. Kantor more than one hundred years ago [Kt]. It was continued by Segre, Manin, Iskovskih, Gizatullin and many others [Se], [Ma1, 2], [Is], [Gi]. See also [Ho1], [Ho2]. In [DO], the group of automorphisms of any general del Pezzo surface is described and it turns out that its discrete part is equal to the kernel of the Cremona representation on the moduli space of $n$ points in $\mathbf{P}^{2}$. Very recently, de Fernex $[\mathrm{dF}]$ constructed all the Cremona transformations of $\mathbf{P}^{2}$ of prime order, where he employed the methods different from those used by Dolgachev and Zhang in [ZD].

In [ZD], minimal pairs $(X, G)$ with prime order $p=|G|$ was considered. In particular, using the recent Mori theory, it was shown there that if the $G$-invariant sublattice of Pic $X$ has rank 1 then $p \leq 5$ unless $X=\mathbf{P}^{2}$; the short and precise classification of these pairs, modulo equivariant isomorphism, was also given there. 
Generic Enriques surfaces have infinitely many automorphisms. Those Enriques surfaces with finite automorphisms have been classified by S. Kondō ([Kon4], see also [Ni2]); there are seven families of such Enriques surfaces. It follows that K3 covers of Enriques surfaces all have infinite automorphism groups.

For K3 surfaces, much progress on their automorphism groups has been done by Kondō, Keum, Dolgachev, Oguiso, and Zhang ([Kon1, 2, 3], [Ke1, 3], [KK], [DK], [OZ1, 3, 4, 5]).

It is known that minimal surfaces of general type has only finite automorphism groups. It was Xiao [Xi1] who gave a proof of the existence of a bound for the order of the automophism group, which is linear in the Euler number of the surface. For curves $C$ of genus $\geq 2$, a classical theorem of Hurwitz gave a sharp bound $|\operatorname{Aut}(C)| \leq 84(g(C)-1)=-42 e(C)$.

Theorem [Xi2, Theorem 2]. Let $X$ be a minimal surface of general type. Then $|\operatorname{Aut}(X)| \leq$ $\left(42 K_{X}\right)^{2}$, with equality if and only if $X \cong(C \times C) / N$, where $C$ is a curve with $\mid$ Aut $(C) \mid=$ $84(g(C)-1), N$ a normal subgroup of $\operatorname{Aut}(C \times C)$ acting freely on $C \times C$ and preserving the two projections of $C \times C$.

(5.2). In [ZD], pairs $(X, G)$ of a smooth rational projective surface $X$ and a finite group of automorphisms are considered. A pair is minimal if every $G$-equivariant birational morphism to another pair $(Z, G)$ is an isomorphism.

Theorem [ZD, Therorem 1]. Let $\left(X, \mu_{p}\right)$ be a minimal pair with $p$ a prime number.

(1) If the invariant sub-lattice $(\operatorname{Pic} X)^{\mu_{p}}$ has rank at least 2 , then $X$ is a Hirzebruch surface and the pair $\left(X, \mu_{p}\right)$ is birationally equivalent to a pair $\left(\mathbf{P}^{2}, \mu_{p}\right)$.

(2) If (Pic $X)^{\mu_{p}}$ has rank 1 , then the pairs are classified in $[Z D$, Theorem 1$]$; in particular, we have $p \leq 5$ unless $X=\mathbf{P}^{2}$.

(5.3). Let $X$ be a K3 surface. The following are well known (cf. [BPV]).

(1) $H^{2,0}(X)=\mathbf{C} \omega_{X}$,

where $\omega_{X}$ is a nowhere vanishing global holomorphic 2 -form on $X$.

(2) $H^{2}(X, \mathbf{Z})$ is an even unimodular lattice of signature $(3,19)$ with the cup product, so we have an isomorphism

$$
H^{2}(X, \mathbf{Z}) \cong U \oplus U \oplus U \oplus E_{8}(-1) \oplus E_{8}(-1),
$$

where $U$ (resp. $E_{8}$ ) is the even unimodular lattice of signature $(1,1)$ (resp. $(8,0)$ ).

(3) $\operatorname{Pic}(X)$ is isomorphic to the Néron-Severi group $N S(X)$, and hence can be viewed as a sublattice of $H^{2}(X, \mathbf{Z})$. The rank of Pic $(X)$, called the Picard number of $X$, is denoted by $\rho(X)$. This number can take the value $0,1, \ldots, 20$. The lattice $\operatorname{Pic}(X)$ is hyperbolic(=Lorentzian) if $X$ is projective, and is semi-negative definite or negative definite if $X$ is not projective. 
(4) Recall that all K3 surfaces are Kähler [Siu], so Hodge decomposition holds for them.

(5) Let

$$
C(X) \subset H^{1,1}(X, \mathbf{R}):=H^{1,1}(X) \cap H^{2}(X, \mathbf{R})
$$

denote the Kähler cone of $X$, the set of all classes of symplectic forms of Kähler-Einstein metrics on $X$. In $\mathrm{K} 3$ surface case, $C(X)$ can be numerically characterized as follows:

$$
C(X)=\left\{\omega \in H^{1,1}(X, \mathbf{R}):\langle\omega, \omega\rangle>0,\langle\omega, R\rangle>0 \text { for all smooth rational curves } R\right\}
$$

For a compact Kähler manifold, Nakai-Moishezon type criterion, i.e. the characterization of the Kähler cone, is highly non-trivial (see [DP]).

(6) Let $r$ be an element of $\operatorname{Pic}(X)$ with $\langle r, r\rangle=-2 d(d>0)$, and $\langle r, \operatorname{Pic}(X)\rangle \subset d \mathbf{Z}$. Then

$$
x \rightarrow x+\langle x, r\rangle r / d
$$

defines an isometry of Pic $(X)$, called a $(-2 d)$-reflection.

Let $W(\operatorname{Pic}(X))\left(\operatorname{resp} . W(\operatorname{Pic}(X))^{(2)}\right)$ be the subgroup of the orthogonal group $O(\operatorname{Pic}(X))$ generated by all reflections (resp. all $(-2)$-reflections). These are normal subgroups of $O(\operatorname{Pic}(X))$ and, by linearity, acts naturally on $H^{1,1}(X, \mathbf{R})$. The set

$$
\left\{\omega \in H^{1,1}(X, \mathbf{R}):\langle\omega, \omega\rangle>0\right\}
$$

has two components, each a cone over a 19-dimensional hyperbolic manifold with constant curvature. $C(X)$ is contained in one of the two components, and the action of $W(\operatorname{Pic}(X))^{(2)}$ on this component has $C(X)$ as its fundamental domain.

(7) If $X$ is projective, the ample cone

$$
D(X):=C(X) \cap \operatorname{Pic}(X) \otimes \mathbf{R}
$$

is non-empty and can be numerically characterized as

$$
D(X)=\{\omega \in \operatorname{Pic}(X) \otimes \mathbf{R}:\langle\omega, \omega\rangle>0,\langle\omega, R\rangle>0 \text { for all smooth rational curves } R\} .
$$

The group $W(\operatorname{Pic}(X))^{(2)}$ acts on the component $P_{+}(X)$ of

$$
\{\omega \in \operatorname{Pic}(X) \otimes \mathbf{R}:\langle\omega, \omega\rangle>0\}
$$

containing $D(X)$, and has $D(X)$ as its fundamental domain.

Note that $O(\operatorname{Pic}(X))$ acts on $P_{+}(X)$ and is a semi-direct product of the normal subgroup $W(\operatorname{Pic}(X))^{(2)}$ and the symmetry group $\operatorname{Sym} D(X)$ of the cone $D(X)$, i.e.

$$
O(\operatorname{Pic}(X)) / W(\operatorname{Pic}(X))^{(2)} \cong \operatorname{Sym} D(X) .
$$


(5.4). The Torelli theorem asserts that a K3 surface is determined up to isomorphism by its Hodge structure. More precisely we have:

Theorem ([PSS],[BR]). Let $X$ and $Y$ be K3 surfaces, and let

$$
\phi: H^{2}(X, \mathbf{Z}) \rightarrow H^{2}(Y, \mathbf{Z})
$$

be an isometry. Extend $\phi$ to $H^{2}(X, \mathbf{C})$ or to $H^{2}(X, \mathbf{R})$ by tensoring with $\mathbf{C}$ or $\mathbf{R}$. Then :

(1) If $\phi$ sends $H^{2,0}(X)$ to $H^{2,0}(Y)$, then $X$ and $Y$ are isomorphic.

(2) If $\phi$ also sends $C(X)$ to $C(Y)$, then $\phi=f^{*}$ for a unique isomorphism $f: Y \rightarrow X$.

(5.5). Let $X$ be a projective K3 surface. Torelli theorem shows that there is a map

$$
\operatorname{Aut}(X) \rightarrow O(\operatorname{Pic}(X)) / W(\operatorname{Pic}(X))^{(2)} \cong \operatorname{Sym} D(X)
$$

which has finite kernel and cofinite image. So in practice, if we want to describe $\operatorname{Aut}(X)$, the main step is to calculate $O(\operatorname{Pic}(X)) / W(\operatorname{Pic}(X))^{(2)}$. This is in general a highly nontrivial arithmetic problem, if the group is infinite. There are 3 cases:

(1) $W(\operatorname{Pic}(X))^{(2)}$ is of finite index in $O(\operatorname{Pic}(X))$.

(2) $W(\operatorname{Pic}(X))^{(2)}$ is of infinite index in $O(\operatorname{Pic}(X))$, but $W(\operatorname{Pic}(X))$ is of finite index in $O(\operatorname{Pic}(X))$. (In this case, we call $\operatorname{Pic}(X)$ reflective.)

(3) $W(\operatorname{Pic}(X))$ is of infinite index in $O(\operatorname{Pic}(X))$, i.e. $\operatorname{Pic}(X)$ is not reflective.

Remark. The case (1) occurs if and only if $\operatorname{Aut}(X)$ is finite. If $\rho(X) \geq 3$, this occurs if and only if $X$ contains at least one but finitely many smooth rational curves. If $\rho(X)=2$, this occurs if and only if $X$ contains a smooth rational curve or an irreducible curve of arithmetic genus 1 , if and only if $\operatorname{Pic}(X)$ represents -2 or 0 [PSS]. Nikulin [Ni1, Ni4] and Vinberg classified all such lattices of rank $\geq 3$ belonging to the case (1). It follows from the classification that every algebraic Kummer surface has an infinite automorphism group (cf. $[\mathrm{Ke} 2]$ ).

The classification of the Néron-Severi lattice is also utilized in [Og1 - Og3], where he has proved the density of the jumping loci of the Picard number of a hyperkähler manifold under small 1-dimensional deformation, where he reveals the structure of hierarchy among all the narrow Mordell-Weil lattices of Jacobian K3 surfaces.

(5.6). For finite groups which can act on a K3 surface, the following results are given by S. Mukai and S. Kondo.

Theorem [Mu],[Kon2]. Let $X$ be a $K 3$ surface and let $G$ be a finite symplectic subgroup of $\operatorname{Aut}(X)$, i.e. $G$ acts trivially on $H^{2,0}(X)$. Then $G$ is isomorphic to a subgroup of the 
Mathieu group $M_{23}$, which has at least five orbits on a set $\Omega$ of 24 elements. In particular, $|G| \leq 960$.

Theorem [Kon3]. The maximum order among all finite groups which can act on a K3 surface is 3840 , and is uniquely realized by the group $(\mathbf{Z} / 2 \mathbf{Z})^{4} \cdot A_{5} \cdot \mathbf{Z} / 4 \mathbf{Z}$ acting on the Kummer surface $K m\left(E_{\sqrt{-1}} \times E_{\sqrt{-1}}\right)$, where $E_{\sqrt{-1}}$ is the elliptic curve with $\sqrt{-1}$ as its fundamental period.

Some projective K3 surfaces, including all algebraic Kummer surfaces and K3 covers of Enriques surfaces, have infinite automorphism groups. Given a projective K3 surface $X$ with $\operatorname{Aut}(X)$ infinite, it is an interesting problem to determine a set of geometric generators of Aut $(X)$. This problem has been settled for certain classes of K3 surfaces. These results are given in (5.7) - (5.10) below.

(5.7). Two most algebraic K3 surfaces

Vinberg[Vin] calculated $\operatorname{Aut}(X)$ for two K3 surface with transcendental lattice

$$
T(X)=\left(\begin{array}{ll}
2 & 1 \\
1 & 2
\end{array}\right), \quad\left(\begin{array}{ll}
2 & 0 \\
0 & 2
\end{array}\right)
$$

respectively. In both cases, the full reflection group $W(\operatorname{Pic}(X))$ is of finite index in $O(\operatorname{Pic}(X))$.

(5.8). generic Jacobian Kummer surfaces

Let $C$ be a smooth curve of genus 2. The Jacobian variety $J(C)$ of $C$ is an abelian surface with a natural involution $\tau$ and the quotient variety $J(C) / \tau$ has 16 singularities of type $A_{1}$. This surface can be embedded as a quartic surface $F$ in $\mathbf{P}^{3}$ with 16 nodes. The minimal resolution $X$ of $J(C) / \tau$ is called the Jacobian Kummer surface associated with $C$. We call $X$ generic if the Néron-Severi group of $J(C)$ is generated by the class of $C$. For $X$ generic, the transcendental lattice $T(X)$ can be computed as follows:

$$
T(X)=U(2) \oplus U(2) \oplus<-4>.
$$

Note that $\operatorname{Aut}(X)$ is isomorphic to the birational automorphism group $\operatorname{Bir}(F)$ of the singular quartic surface $F$. At the last century it was known that $X$ has many involutions, that is, sixteen translations induced by those of $J(C)$ by a 2-torsion point, sixteen projections of $F$ from a node, sixteen correlations by means of the tangent plane collinear to a trope, and a switch defined by the dual map of $F$. In 1900, Hutchinson found another 60 involutions associated with Göpel tetrads. Since Hutchinson, for generic $X$ no other automorphism had been provided until new 192 automorphisms were given in [Ke1].

Theorem [Ke1]. For a generic Jacobian Kummer surface, there are 192 new automorphisms of infinite order which are not generated by classical involutions. 
Theorem [Kon1]. The automorphism group of a generic Jacobian Kummer surface is generated by the classical involutions and the 192 new automorphisms.

Theorem [Ke3]. For F generic, all birational automorphisms of $F$ are induced by Cremona transformations of $\mathbf{P}^{3}$.

(5.9). Kummer surfaces associated with the product of two elliptic curves

The following four cases were considered. In each case, a set of generators of $\operatorname{Aut}(X)$ is given in $[\mathrm{KK}]$.

Case I. $X=K m(E \times F)$ where $E$ and $F$ are non-isogenus generic elliptic curves.

Case II. $X=K m(E \times E)$ where $E$ is an elliptic curve without complex multiplications.

Case III. $X=K m\left(E_{\omega} \times E_{\omega}\right)$ where $\omega$ is a 3rd root of unity and $E_{\tau}$ is the elliptic curve with $\tau$ as its fundamental period.

Case IV. $X=K m\left(E_{\sqrt{-1}} \times E_{\sqrt{-1}}\right)$.

The transcendental lattice $T(X)$ can be computed as follows:

$$
T(X)= \begin{cases}U(2) \oplus U(2), & (\text { Case I) } \\
U(2) \oplus<4>, & (\text { Case II); } \\
\left(\begin{array}{ll}
4 & 2 \\
2 & 4
\end{array}\right), & (\text { Case III) } \\
\left(\begin{array}{ll}
4 & 0 \\
0 & 4
\end{array}\right), & \text { (Case IV) }\end{cases}
$$

Remark. In Case I the group $W(\operatorname{Pic}(X))$ is of finite index in $O(\operatorname{Pic}(X))$ and in other cases not.

(5.10). Quartic Hessian surfaces

Let $S: F\left(x_{0}, x_{1}, x_{2}, x_{3}\right)=0$ be a nonsingular cubic surface in $\mathbf{P}^{3}$. Its Hessian surface is a quartic surface defined by the determinant of the matrix of second order partial derivatives of the polynomial $F$. When $F$ is general enough, the quartic $H$ is irreducible and has 10 nodes. It contains also 10 lines which are the intersection lines of five planes in general linear position. The union of these five planes is classically known as the Sylvester pentahedron of $S$. The equation of $S$ can be written as the sum of cubes of some linear forms defining the five planes. A nonsingular model of $H$ is a K3 surface $\tilde{H}$. Its Picard number $\rho$ satisfies the inequality $\rho \geq 16$. Note that $\operatorname{Aut}(\tilde{H}) \cong \operatorname{Bir}(H)$. In $[\mathrm{DK}]$ an explicit description of the group $\operatorname{Bir}(H)$ is given when $S$ is general enough so that $\rho=16$. In this general case, the transcendental lattice $T(\tilde{H})$ can be computed as follows:

$$
T(\tilde{H})=U \oplus U(2) \oplus A_{2}(-2) .
$$


Although $H$, in general, does not have any non-trivial automorphisms (because $S$ does not), the group $\operatorname{Bir}(H) \cong \operatorname{Aut}(\tilde{H})$ is infinite. It is generated by the automorphisms defined by projections from the nodes of $H$, a birational involution which interchanges the nodes and the lines, and the inversion automorphisms of some elliptic pencils on $\tilde{H}$.

This can be compared with the known structure of the group of automorphisms of the Jacobian Kummer surface (5.8). Indeed, the latter surface is birationally isomorphic to the Hessian $H$ of a cubic surface [Hu] but the Picard number of $\tilde{H}$ is equal to 17 instead of 16 .

(5.11). Let us explain the method for computing the automorphism group of an algebraic K3 surface, which was first employed by S. Kondō for generic Jacobian Kummer surface case (5.8) [Kon1]. The two cases (5.9) and (5.10) use this method, and even the first case (5.7) can also be calculated by the same method (See [Bor2]).

Let $X$ be an algebraic K3 surface with large Picard number, say $\rho(X) \geq 3$. Suppose that $\operatorname{Aut}(X)$ is infinite. Then the ample cone $D(X)$ is not a (finite) polyhedral cone, i.e. has infinitely many faces. Hence, it is difficult to describe $D(X)$ explicitly. Assume that one can find

- a polyhedral cone $D^{\prime}$ in $D(X)$,

- a set of automorphisms $\left\{g_{\alpha}\right\}$ of $X$ whose action on $D(X)$ has $D^{\prime}$ as a fundamental domain.

Then, by (5.5), one can conclude that the automorphisms $\left\{g_{\alpha}\right\}$ generate the whole group $\operatorname{Aut}(X)$, up to finite groups. In addition to $\left\{g_{\alpha}\right\}$, some symmetries of $D^{\prime}$ (not all elements of $S y m D^{\prime}$ in general) may realize as automorphisms of $X$ and some projectively linear automorphisms, if any, generate the kernel of the map in (5.5).

Remark. In the above, $g_{\alpha}$ corresponds to a face of $D^{\prime}$ orthogonal to a vector $\alpha$, and acts on $D(X)$ like a reflection, i.e. sends one of the half-spaces defined by $\alpha$ to the other half-space defined by $\alpha$ or to one of the two half-spaces corresponding to $g_{\alpha}^{-1}$. The second case actually occurs in generic Jacobian Kummer surface case (5.8).

(5.12). To find such a polyhedral cone $D^{\prime} \subset D(X)$, Kondō used the known structure of the orthogonal group of the even unimodular lattice $I I_{1,25}$ of signature $(1,25)$. (Such a lattice is unique up to isomorphism and is isomorphic to $\Lambda \oplus U$, where $\Lambda$ is the Leech lattice, i.e. the even unimodular negative definite lattice of rank 24 which contains no vectors of norm -2.) To be more precise, the following steps lead to the calculation of $\operatorname{Aut}(X)$.

Step 1. Compute Pic $(X)$.

Step 2. Embed Pic $(X)$ primitively into $I I_{1,25}=\Lambda \oplus U$ such that the projection of the 
Weyl vector $w=(0,(0,1)) \in \Lambda \oplus U$ onto Pic $(X) \otimes \mathbf{R}$ must be an ample class.

Conway [Co] described a fundamental domain $D$ of the reflection group $W\left(I I_{1,25}\right)^{(2)}$ in terms of the Leech roots (=roots with intersection number 1 with the Weyl vector $w$ ). More precisely, he showed that $W\left(I I_{1,25}\right)^{(2)}$ is generated by $(-2)$-reflections corresponding to Leech roots.

Step 3. The fundamental domain $D$ of the reflection group $W\left(I I_{1,25}\right)^{(2)}$ cuts out a finite polyhedral cone $D^{\prime}$ inside the ample cone $D(X)$. In other words,

$$
D^{\prime}=D \cap P_{+}(X),
$$

where $P_{+}(X)$ is the positive component of $\{\omega \in \operatorname{Pic}(X) \otimes \mathbf{R}:\langle\omega, \omega\rangle>0\}$. Indeed, $D$ contains the Weyl vector $w$ and, by Step 2, the projection of $w$ is contained in $D^{\prime}$.

Determine the hyperplanes $\alpha$ which bound $D^{\prime}$. The reason why $D^{\prime}$ is polyhedral comes from Borcherds [Bor1]; among infinitely many faces of $D$, those intersecting $P_{+}(X)$ bound $D^{\prime}$, and these faces correspond to Leech roots having a non-zero projection onto Pic $(X) \otimes \mathbf{R}$.

Step 4. Match the faces $\alpha$ of $D^{\prime}$ with automorphisms $g_{\alpha}$ such that $g_{\alpha}$ sends one of the half-spaces defined by $\alpha$ to the other half-space defined by $\alpha$ or to one of the two half-spaces corresponding to $g_{\alpha}^{-1}$.

This allows one to prove that the automorphisms $g_{\alpha}$ generate a group of symmetries of $D(X)$, having $D^{\prime}$ as its fundamental domain.

Step 5. Take care of $S y m D^{\prime}$. See if which symmetries of $D^{\prime}$ realize as automorphisms of $X$. Finally see if there are any projectively linear automorphisms, (which generate the kernel of the map in (5.5)).

Remark. In the known cases (5.7) - (5.10), the embedding $\operatorname{Pic}(X) \subset \Lambda \oplus U$ is given in such a way that $\operatorname{Pic}(X)$ is the orthogonal complement of a root sublattice of $\Lambda \oplus U$. For example, in case (5.7) the orthogonal complement of $\operatorname{Pic}(X)$ in $\Lambda \oplus U$ is a primitive sublattice of rank 10 which contains a negative definite root lattice of type $A_{5}+A_{1}^{5}$, which is of index 2 .

In practice, Step 4 seems most complicated. The automorphism $g_{\alpha}$ works like a reflection, but is not necessarily an involution. It may be of infinite order. At any rate, $g_{\alpha}$ may be geometrically evident, or can be picked up from a list of already known automorphisms, or may be found by looking at extra structures of $X$, e.g. elliptic fibrations, double plane structures,..., etc. In worst cases, one has to find an (abstract) effective Hodge isometry of $H^{2}(X, \mathbf{Z})$ and then realize it geometrically.

The reason why the beautiful combinatorics of the Leech lattice plays a role in the description of the automorphism groups of K3 surfaces is still unclear to us. We hope that the classification of all K3 surfaces whose Picard lattice is isomorphic to the orthogonal complement of a root sublattice of $I I_{1,25}$ will shed more light to this question. 
Remark (5.13). The method (5.12) also works for some supersingular K3 surfaces. I. Dolgachev and S. Kondō [DoKo] have computed the automorphism group of a supersingular $\mathrm{K} 3$ surface in characteristic 2 whose Picard lattice is $U \oplus D_{20}$. (For supersingular K3 surfaces Torelli type theorem holds, i.e. an automorphism of a supersingular K3 surface is determined by its action on the Picard lattice.)

An even lattice $L$ is reflective if its reflection group $W(L)$ is of finite index in $O(L)$ (see (5.5)). The lattice $U \oplus D_{20}$ is reflective as it is pointed out by Borcherds [Bor1] and it is the only known example, up to scaling, of an even reflective hyperbolic lattice of rank 22. The range of possible rank of an even reflective lattice of signature $(1, r-1), r \geq 1$, is given by Esselmann [Es]: it takes the same range 1,2,.., 20, 22 as the Picard number of a $\mathrm{K} 3$ surface in positive characteristic.

\section{Section 6. Automorphisms of Algebraic surfaces - Singular surface CASE}

(6.1). In [MM] and [MZ3], one considers pairs $(V, G)$ of surface $V$ and group $G$ of automorphisms, where $V$ may be singular and even non-complete.

(6.2). Let $Y$ be a Gorenstein del Pezzo singular surface of Picard number 1. In [Z9], one classifies all actions on $Y$ by cyclic groups $\mathbf{Z} /(p)$ of prime order $p \geq 5$.

Theorem [Z9, Theorems A and C].

Let $Y$ be a Gorenstein del Pezzo singular surface of Picard number 1. Then we have:

(1) Either $|\operatorname{Aut}(Y)|=2^{a} 3^{b}$ for some $1 \leq a+b \leq 7$, or $\operatorname{Aut}(Y) \supseteq \mathbf{Z} /(p)$ for every prime $p \geq 5$ and hence $|\operatorname{Aut}(Y)|=\infty$.

(2) $|\operatorname{Aut}(Y)|$ is finite if and only if either $\operatorname{Sing} Y=A_{7}$ or $K_{Y}^{2}=1$ and $\left|-K_{Y}\right|$ has at least three singular members.

(3) Let $p \geq 5$ be a prime. Suppose that $Y$ is not isomorphic to the quadric cone in $\mathbf{P}^{3}$. Then modulo equivariant isomorphism, there is either none, or only one, or exactly $p+1$ action(s) of $\mathbf{Z} /(p)$ on $Y$. All actions are given in $[Z 9]$.

Remark 6.3. We like to compare (6.2) with known results for smooth del Pezzo surfaces. (1) If $X$ is a generic rational surface with $K_{X}^{2} \leq 5$, then $|\operatorname{Aut}(X)|$ divides 5! (see [DO], $[\mathrm{Ki}])$.

(2) If $X$ is a del Pezzo surface of degree 3, 4, then $\operatorname{Aut}(X)$ does not contains $\mathbf{Z} /(p)$ for any prime $p \geq 7$, and modulo equivariant isomorphism there is at most one non-trivial $\mathbf{Z} /(5)$ action on $X[\mathrm{Ho} 1,2]$.

(3) Let $X$ be a rational projective surface with a non-trivial $\mathbf{Z} /(p)$-action for some prime $p$ such that the $\mathbf{Z} /(p)$-invariant sublattice of $\operatorname{Pic} X$ is of rank 1 (this condition is automatic if the Picard number $\rho(X)=1$ ). If $X$ is smooth, then $p \leq 5$ unless $X=\mathbf{P}^{2}$ [ZD, Theorem 1].

\section{ACKNOWLEDGEMENT}

We would like to thank the referee for careful reading and suggestions which improve the 
paper. This article was initiated when both of us were attending the conference - Algebraic Geometry in East Asia - in August 2001, and we would like to thank the organizers for providing the wonderful environment and the encouragement for us to write a survey article. This work was finalized when the second-named author visited Korea Institute for Advanced Study in December 2001, and he likes to thank the institute for the hospitality. This work was partially supported by Korea Science and Engineering Foundation (R011999-00004) and an Academic Research Fund of National University of Singapore.

\section{REFERENCES}

[AN] V. A. Alekseev and V. V. Nikulin, Classification of del Pezzo surfaces with logterminal singularities of index $\leq 2$ and involutions on $K 3$ surfaces, Soviet Math. Dokl. 39 (1989), 525-528.

[Ar1] M. Artin, Some numerical criteria for contractability of curves on algebraic surfaces. Amer. J. Math. 84 (1962), 485-496.

[Ar2] M. Artin, On isolated rational singularities of surfaces, Amer. J. Math. 88 (1966), $129-136$.

[BPV] W. Barth, C. Peters and A. Van de Ven, Compact Complex Surfaces, SpringerVerlag, 1984.

[Bl] R. Blache, The structure of l.c. surfaces of Kodaira dimension zero, I, J. Algebraic Geom. 4 (1995), 137-179.

[Bor1] R. Borcherds, Automorphism groups of Lorentzian lattices, J. Algebra 111 (1987), 133-153.

[Bor2] R. Borcherds, Coxter groups, Lorentzian lattices, and K3 surfaces, International Mathematics Research Notices 19 (1998), 1011-1031.

[Br] E. Brieskorn, Rationale Singularitäten komplexer Flächen, Invent. Math. 4 (1967 / 1968), 336-358.

[BR] D. Burns, Jr. and M. Rapoport, On the Torelli theorem for Kählerian $K 3$ surfaces, Ann. Sci. Ecole Norm. Sup. (4) 8 (1975), 235-273.

[Ca] F. Campana, Connexiti rationnelle des variitis de Fano, Ann. Sci. Ecole Norm. Sup. 25 (1992), 539-545.

[CKO] F. Catanese, J. Keum and K. Oguiso, Some remarks on the universal cover of an open K3 surface, Math. Ann. to appear, math.AG/0104015.

[Co] J.H. Conway, The automorphism group of the 26 dimensional even Lorentzian lattice, J. Algebra 80 (1983), 159-163.

[CD] F. Cossec and I. V. Dolgachev, Enriques surfaces, I, Progress in Mathematics, 76 (1989), Birkhäuser Boston, Inc.

$[\mathrm{dF}]$ Tommaso de Fernex, Birational trasformations of prime order of the projective plane, preprint 2001.

[DP] J. -P. Demailly and M. Paun, Numerical characterization of the Kähler cone of a compact Kähler manifold, math.AG / 0105176.

[DK] I. V. Dolgachev and J. Keum, Birational automorphisms of quartic Hessian surfaces, Trans. Amer. Math. Soc. 354 (2002), 3031-3057. 
[DoKo] I. V. Dolgachev and S. Kondō, A supersingular K3 surface in characteristic 2 and the Leech lattice, math.AG/0112283 v2.

[DO] I. Dolgachev and D. Ortland, Point sets in projective spaces and theta functions, Astrisque, Vol. 165 (1988).

[DZ] I. V. Dolgachev and D. -Q. Zhang, Coble rational surfaces, Amer. J. Math. 123 (2001), 79-114.

[Du] A. H. Durfee, Fifteen characterizations of rational double points and simple critical points, Enseign. Math. 25 (1979), 131-163.

[Es] F. Esselmann, Ueber die maximale Dimension von Lorenz-Gittern mit coendlicher Spiegelungsgruppe, J. Number Theory, 61 (1996), 103-144.

[FKL] A. Fujiki, R. Kobayashi and S. Lu, On the fundamental group of certain open normal surfaces. Saitama Math. J. 11 (1993), 15-20.

[Fu] T. Fujita, On the topology of noncomplete algebraic surfaces, J. Fac. Sci. Univ. Tokyo Sect. IA Math. 29 (1982), 503-566.

[Gi] M. H. Gizatullin, Rational G-surfaces, Math. USSR Izv. 16 (1981), 103-134.

[GHS] T. Graber, J. Harris and J. Starr, Families of rationally connected varieties, math.AG / 0109220.

[GPa] R. V. Gurjar and A.J. Parameswaran, Affine lines on Q-homology planes, J. of Math. Kyoto Univ. 35 (1995), 63-77.

[GPr] R. V. Gurjar and C. R. Pradeep, $Q$-homology planes are rational, III, Osaka J. Math. 36 (1999), 259-335.

[GPS] R. V. Gurjar, C. R. Pradeep and A. R. Shastri, On rationality of logarithmic Qhomology planes, II. Osaka J. Math. 34 (1997), 725-743.

[GS] R. V. Gurjar and A. R. Shastri, On the rationality of complex homology 2-cells, I; II, J. Math. Soc. Japan, 41 (1989), 37-56; 41 (1989), 175-212.

[GZ1] R. V. Gurjar and D. -Q. Zhang, $\pi_{1}$ of smooth points of a log del Pezzo surface is finite, I, J. Math. Sci. Univ. Tokyo 1 (1994), 137-180.

[GZ2] R. V. Gurjar and D. -Q. Zhang, $\pi_{1}$ of smooth points of a log del Pezzo surface is finite, II, J. Math. Sci. Univ. Tokyo 2 (1995), 165-196.

[GZ3] R. V. Gurjar, and D. -Q. Zhang, Normal algebraic surfaces with trivial bicanonical divisor, J. Algebra 186 (1996), 970-989.

[Ho1] T. Hosoh, Automorphism groups of cubic surfaces, J. Algebra 192 (1997), 651-677.

[Ho2] T. Hosoh, Automorphism groups of quartic del Pezzo surfaces, J. Algebra 185 (1996), 374-389.

[Hu] J. Hutchinson, The Hessian of the cubic surface, Bul. Amer. Math. Soc. 5 (1889), 282-292.

[I1] S. Iitaka, On logarithmic K3 surfaces, Osaka J. Math. 16 (1979), 675-705.

[I2] S. Iitaka, A numerical criterion of quasi-abelian surfaces, Nagoya Math. J. 73 (1979), 99-115.

[I3] S. Iitaka, Algebraic geometry - An introduction to birational geometry of algebraic varieties, Graduate Texts in Mathematics, 76, Springer-Verlag, New York-Berlin, 1982. 
[Is] V. A. Iskovskih, Minimal models of rational surfaces over arbitrary fields, Math. USSR Izv. 14 (1981), 17-39.

[Kt] S. Kantor, Theorie der endlichen Gruppen von eindeutigen Transformationen in der Ebene, Berlin: Mayer \& Muller, 1895.

[KN] M. Kato and I. Naruki, Depth of rational double points on quartic surfaces, Proc. Japan Acad. Ser. A Math. Sci. 58 (1982), 72-75.

[Ka1] Y. Kawamata, On the classification of noncomplete algebraic surfaces, Lecture Notes in Math. 732 (1979), 215-232. Springer, Berlin.

[Ka2] Y. Kawamata, The cone of curves of algebraic varieties, Ann. of Math. 119 (1984), 603-633.

[KMM] Y. Kawamata, K. Matsuda and K. Matsuki, Introduction to the minimal model problem, Adv. Stud. Pure Math. 10 (1987), 283-360.

[KMc] S. Keel and J. McKernan, Rational curves on quasi-projective surfaces, Mem. Amer. Math. Soc. 140 (1999), no. 669.

[KMo] J. Kollar and S. Mori, Birational geometry of algebraic varieties, Cambridge Tracts in Mathematics, 134 (1998).

[Ke1] J. Keum, Automorphisms of Jacobian Kummer surfaces, Compositio Math. 107 (1997), 269-288.

[Ke2] J. Keum, Every algebraic Kummer surface has infinitely many automorphisms, unpublished manuscript (1996).

[Ke3] J. Keum, Automorphisms of a generic Jacobian Kummer surface, Geom. Ded. 76 (1999), 177-181.

[KK] J. Keum and S. Kondo, The automorphism groups of Kummer surfaces associated with the product of two elliptic curves, Trans. Amer. Math. Soc. 353 (2001), 1469-1487.

[KZ] J. Keum and D. -Q. Zhang, Fundamental groups of open K3 surfaces, Enriques surfaces and Fano 3-folds, J. Pure App. Alg. 170 (2002), 67-91.

[Kb1] R. Kobayashi, Uniformization of complex surfaces. Kähler metric and moduli spaces, Adv. Stud. Pure Math. 18-II (1990), 313-394.

[Kb2] R. Kobayashi, Einstein-Kähler $V$-metrics on open Satake $V$-surfaces with isolated quotient singularities, Math. Ann. 272 (1985), 385-398.

[KNS] R. Kobayashi, S. Nakamura and F. Sakai, A numerical characterization of ball quotients for normal surfaces with branch loci, Proc. Japan Acad. Ser. A Math. Sci. 65 (1989), 238-241.

[Ki] M. Koitabashi, Automorphism groups of generic rational surfaces, J. Algebra 116 (1988), $130-142$.

[Kj1] H. Kojima, Open rational surfaces with logarithmic Kodaira dimension zero, Internat. J. Math. 10 (1999), 619-642.

[Kj2] H. Kojima, Logarithmic del Pezzo surfaces of rank one with unique singular points, Japan. J. Math. (N.S.) 25 (1999), 343-375.

[Kj3] H. Kojima, Complements of plane curves with logarithmic Kodaira dimension zero, J. Math. Soc. Japan 52 (2000), 793-806. 
[Kj4] H. Kojima, Rank one log del Pezzo surfaces of index two, J. Math. Kyoto Univ. to appear.

[KoMiMo] J. Kollar, Y. Miyaoka and S. Mori, Rationally connected varieties, J. Alg. Geom. 1 (1992), 429-448.

[Kon1] S. Kondō, The automorphism group of a generic Jacobian Kummer surface, J. Alg. Geom. 7 (1998), 589-609.

[Kon2] S. Kondō, Niemeier lattices, Mathieu groups, and finite groups of symplectic automorphisms of K3 surfaces (with an appendix by S. Mukai), Duke Math. J. 92 (1998), 593-603.

[Kon3] S. Kondō, The maximum order of finite groups of automorphisms of $K 3$ surfaces, Amer. J. Math. 121 (1999), 1245-1252.

[Kon4] S. Kondō, Enriques surfaces with finite automorphism groups, Japan. J. Math. 12 (1986), 191-282.

[Ma1] Yu. I. Manin, Rational surfaces over perfect fields, II, Math. USSR Sb. 1 (1967), 141-168.

[Ma2] Yu. I. Manin, Cubic forms : Algebra, geometry, arithmetic, 2nd ed, North-Holland Math. Library, 4 (1986), North-Holland Publ. Co., Amsterdam-New York.

[Mi] M. Miyanishi, Open algebraic surfaces, CRM Monograph Series, 12, American Mathematical Society, 2001.

$[\mathrm{MM}]$ M. Miyanishi and K. Masuda, Open algebraic surfaces with finite group actions, Transform. Group, to appear.

[MT1] M. Miyanishi and S. Tsunoda, Noncomplete algebraic surfaces with logarithmic Kodaira dimension $-\infty$ and with nonconnected boundaries at infinity, Japan. J. Math. (N.S.) 10 (1984), 195-242.

[MT2] M. Miyanishi and S. Tsunoda, Logarithmic del Pezzo surfaces of rank one with noncontractible boundaries, Japan. J. Math. (N.S.) 10 (1984), 271-319.

[MZ1] M. Miyanishi and D. -Q. Zhang, Gorenstein log del Pezzo surfaces of rank one, J. Algebra 118 (1988), 63-84.

[MZ2] M. Miyanishi and D. -Q. Zhang, Gorenstein log del Pezzo surfaces, II, J. Algebra 156 (1993), 183-193.

[MZ3] M. Miyanishi and D. -Q. Zhang, Equivariant classification of Gorenstein open log del Pezzo surfaces with finite group actions, Preprint 2001.

[Mu] S. Mukai, Finite groups of automorphisms of $K 3$ surfaces and the Mathieu group, Invent. Math. 94 (1988), 183-221.

[Ni1] V.V. Nikulin, On the quotient groups of the automorphism group of hyperbolic forms by the subgroups generated by 2-reflections, J. Soviet Math. 22 (1983), 1401-1476.

[Ni2] V.V. Nikulin, On the description of groups of automorphisms of Enriques surfaces, Soviet Math. Dokl. 277 (1984), 1324-1327.

[Ni3] V.V. Nikulin, Del Pezzo surfaces with log-terminal singularities, I; II; III, Math. USSR-Sb. 66 (1990), 231-248; Math. USSR-Izv. 33 (1989), 355-372; Math. USSR-Izv. 35 (1990), 657-675. 
[Ni4] V. V. Nikulin, Discrete reflection groups in Lobachevsky spaces and algebraic surfaces, Proc. Intern. Cong. Math. Berkeley, Calif. 1986, pp.654-671.

[Og1] K. Oguiso, Picard numbers in a family of hyperkähler manifolds and applications, J. Alg. Geom. to appear

[Og2] K. Oguiso, Picard numbers in a family of hyperkähler manifolds - A supplement to the article of R. Borcherds, L. Katzarkov, T. Pantev, N. I. Shepherd-Barron, math.AG / 0011258.

[Og3] K. Oguiso, Automorphism groups in a family of K3 surfaces, math.AG / 0104049. [OZ1] K. Oguiso and D. -Q. Zhang, On the most algebraic K3 surfaces and the most extremal log Enriques surfaces, Amer. J. Math. 118 (1996), 1277-1297.

[OZ2] K. Oguiso and D. -Q. Zhang, On the complete classification of extremal log Enriques surfaces, Math. Z. 231 (1999), 23-50.

[OZ3] K. Oguiso and D. -Q. Zhang, K3 surfaces with order 11 automorphisms, math.AG / 9907020.

[OZ4] K. Oguiso and D. -Q. Zhang, On Vorontsov's theorem on $K 3$ surfaces with nonsymplectic group actions, Proc. Amer. Math. Soc. 128 (2000), 1571-1580.

[OZ5] K. Oguiso and D. -Q. Zhang, The Simple Group of Order 168 and K3 Surfaces, in : Complex Geometry, Collection of papers dedicated to Hans Grauert; Bauer, Catanese, Kawamata, Peternell and Siu (ed), Springer, 2002; math.AG / 0011259.

[Oh] K. Ohno, Toward determination of the singular fibers of minimal degeneration of surfaces with $\kappa=0$, Osaka J. Math. 33 (1996), 235-305.

[PSS] I. Piatetski-Shapiro, I.R. Shafarevich, A Torelli theorem for algebraic surfaces of type K3, Math. USSR Izv. 5 (1971), 547-587.

[PS] C. R. Pradeep and A. R. Shastri, On rationality of logarithmic Q-homology planes, I, Osaka J. Math. 34 (1997), 429-456.

[Re1] M. Reid, Young person's guide to canonical singularities, Proc. Sympos. Pure Math. Part 146 (1987), 345-414.

[Re2] M. Reid, Campedelli versus Godeaux - Problems in the theory of surfaces and their classification, Sympos. Math., XXXII (1991), 309-365.

[Sa] F. Sakai, Semistable curves on algebraic surfaces and logarithmic pluricanonical maps, Math. Ann. 254 (1980), 89-120.

[Se] B. Segre, The Non-singular cubic surfaces, Oxford University Press, Oxford, 1942.

[SZ] I. Shimada and D. -Q. Zhang, Classification of extremal elliptic $K 3$ surfaces and fundamental groups of open $K 3$ surfaces, Nagoya Math. J. 161 (2001), 23-54.

[Su] K. Suzuki, On Morrison's cone conjecture for Klt surfaces with $K_{X} \equiv 0$, Comment. Math. Univ. St. Paul 50 (2001), 173-180.

[Siu] Y. T. Siu, Every K3 surface is Kähler, Invent. Math. 73 (1983), 139-150.

[Ta] S. Takayama, Simple connectedness of weak Fano varieties, J. Algebraic Geom. 9 (2000), 403-407.

[Ts] S. Tsunoda, Structure of open algebraic surfaces, I, J. Math. Kyoto Univ. 23 (1983), $95-125$. 
[TZ] S. Tsunoda and D. -Q. Zhang, Noether's inequality for noncomplete algebraic surfaces of general type, Publ. Res. Inst. Math. Sci. 28 (1992), 21-38.

[Vin] E.B. Vinberg, The two most algebraic K3 surfaces, Math. Ann. 265 (1985), 1-21.

[Xi1] G. Xiao, Bound of automorphisms of surfaces of general type. I, Ann. of Math. (2) 139 (1994), no. 1, 51-77.

[Xi2] G. Xiao, Bound of automorphisms of surfaces of general type. II, J. Algebraic Geom. 4 (1995), no. 4, 701-793.

[Z1] D. -Q. Zhang, On Iitaka surfaces, Osaka J. Math. 24 (1987), 417-460.

[Z2] D. -Q. Zhang, Logarithmic del Pezzo surfaces of rank one with contractible boundaries, Osaka J. Math. 25 (1988), 461-497.

[Z3] D. -Q. Zhang, Logarithmic del Pezzo surfaces with rational double and triple singular points, Tohoku Math. J. 41 (1989), 399-452.

[Z4] D. -Q. Zhang, Logarithmic Enriques surfaces, I; II, J. Math. Kyoto Univ. 31 (1991), 419-466; 33 (1993), 357-397.

[Z5] D. -Q. Zhang, Noether's inequality for noncomplete algebraic surfaces of general type, II, Publ. Res. Inst. Math. Sci. 28 (1992), 679-707.

[Z6] D. -Q. Zhang, Algebraic surfaces with nef and big anti-canonical divisor, Math. Proc. Cambridge Philos. Soc. 117 (1995), 161-163.

[Z7] D. -Q. Zhang, The fundamental group of the smooth part of a log Fano variety, Osaka J. Math. 32 (1995), 637-644.

[Z8] D. -Q. Zhang, Algebraic surfaces with log canonical singularities and the fundamental groups of their smooth parts, Trans. Amer. Math. Soc. 348 (1996), 4175-4184.

[Z9] D. -Q. Zhang, Automorphisms of finite order on Gorenstein del Pezzo surfaces, Trans. Amer. Math. Soc. 354 (2002), 4831-4845.

[ZD] D. -Q. Zhang, Automorphisms of finite order on rational surfaces, with an appendix by I. Dolgachev, J. Algebra 238 (2001),560-589.

\section{J. Keum}

Korea Institute for Advanced Study

207-43 Cheongryangri-dong, Dongdaemun-gu

Seoul 130-012, Korea

E-mail : jhkeum@kias.re.kr

D. -Q. Zhang

Department of Mathematics

National University of Singapore

2 Science Drive 2, Singapore 117543

Republic of Singapore

E-mail: matzdq@math.nus.edu.sg 